Zbl. Bakt. Hyg., I. Abt. Orig. C 3, 161-170 (1982)

Lehrstuhl für Biochemie I der Universität Regensburg, Federal Republic of Germany

\title{
The Cell Wall Glycoprotein of Halobacteria: Structural, Functional and Biosynthetic Aspects $\star$
}

\author{
FELIX WIELAND, JOHANN LECHNER, and MANFRED SUMPER
}

Received September 11, 1981

\section{Summary}

The cell wall glycoprotein of Halobacteria contains sulphate covalenty bound to different types of saccharides. A high molecular weight saccharide (HMW-saccharide), which is $\mathrm{N}$-glycosidically linked to Asn was found to have the following composition: $1 \mathrm{gal}$ : 1 galN: 1 glcN : 2 galUA: $2 \mathrm{SO}_{4}{ }^{2-}$. This $\mathrm{HMW}$-saccharide is not a heterosaccharide, but exhibits rather a repeating unit structure similar to that of the animal glycosaminoglycans. Incorporation into the cell wall glycoprotein of this HMW-saccharide is specifically inhibited by the antibiotic bacitracin (Wieland et al., 1980). This inhibition results in a remarkable change of the halobacterial shape: the normally rod shaped cells grown in the presence of bacitracin convert to regular spheres (Mescher and Strominger, 1975).

This HMW-saccharide is synthesized bound to a lipid anchor: only after completion the fully sulphated saccharide is transferred to the core protein.

Key words: Halobacterial glycoprotein - Sulphated glycoconjugates - Glycoprotein biosynthesis - Lipid linked precursor saccharide

\section{Introduction}

Halobacteria were the first bacteria found to possess a true glycoprotein, which is the main constituent of the Halobacterial cell wall (Mescher and Strominger, 1974). This glycoprotein contains saccharides bound by the most common types of linkage known for eucaryotes (c.f. Fig. 1): oligosaccharides free of amino sugars which are O-glycosidically bound to threonine and one large amino sugar contain-

* Paper given at the First International Workshop on Archaebacteria, München, June 27 to July 1, 1981.

Abbreviations: HMW-saccharides: high molecular weight saccharides, SDS gel electrophoresis: dodecylsulphate polyacrylamide gel electrophoresis,

galN : 2-deoxy 2-aminogalactose, glcN: 2-deoxy 2-aminoglucose. 


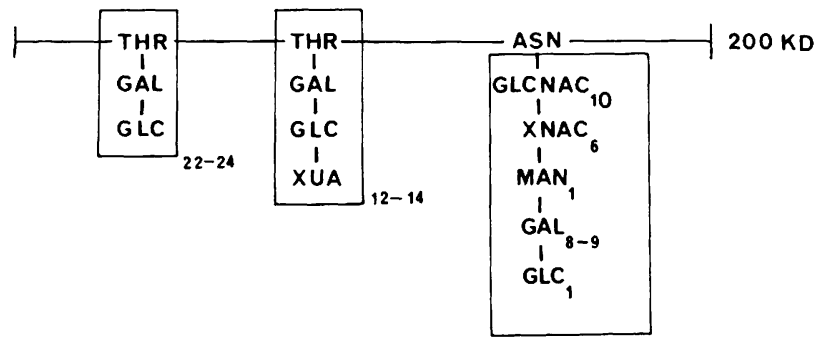

Fig. 1. Structure of the Halobacterium salinarium cell wall glycoprotein as proposed by Mescher and Strominger (1976).

ing saccharide $\mathrm{N}$-glycosidically bound to asparagine which has been depicted as heterosaccharide (Mescher and Strominger, 1976).

In the course of investigations on membrane biosynthesis in Halobacterium halobium we observed that this organism is able to incorporate sulphate into macromolecules without reducing the sulphate residue to cycsteine or methionine (Sumper and Herrmann, 1978). The main product bearing sulphate turned out to be the Halobacterial cell wall glycoprotein (Wieland et al., 1980). In the following the isolation and characterization of sulphated glycopeptides from the Halobacterial cell wall glycoprotein will be described and the function of a sulphated saccharide containing amino sugars will be discussed. Furthermore we will show that this saccharide is synthesized as a lipid-linked precursor and is transferred "en block" to the core protein only after synthesis (including sulphation) is finished.

\section{Materials and Methods}

Most of the materials and methods used in the experiments presented here were described by Wieland et al., 1980 and Wieland et al., 1981. As yet unpublished experimental details will be given in the legends under the appropriate figures.

\section{Results}

\section{Composition of glycopeptides found in the cell wall glycoprotein}

Isolated ${ }^{35} \mathrm{SO}_{4}{ }^{2-}$-labelled Halobacterial cell wall glycoprotein was exhaustively digested with Pronase and a glycopeptide fraction was obtained after chromatography on Dowex AG W $\mathrm{H}^{+}$ion exchange resin in $\mathrm{H}_{2} \mathrm{O}$ in a yield of about 90 to $95 \%$ of the radioactivity. This mixture of glycopeptides was further purified by chromatography on Bio Gel P 10 . Each fraction was assayed for ${ }^{35} \mathrm{SO}_{4}{ }^{2-}$-radioactivity, for neutral sugars and for uronic acids. Fig. 2 shows the corresponding elution profile: with the void volume of the column a radioactive peak appears which contains both neutral sugars und uronic acids. Amino sugar analysis showed a hexosamine and glcN in a $1: 1$ stoichiometry. The hexosamine could be demonstrated to be galN by combined gas liquid chromatography - mass spectrometry. For this analysis we are indepted to Dr. Schäfer, Martinsried. This high molecular 


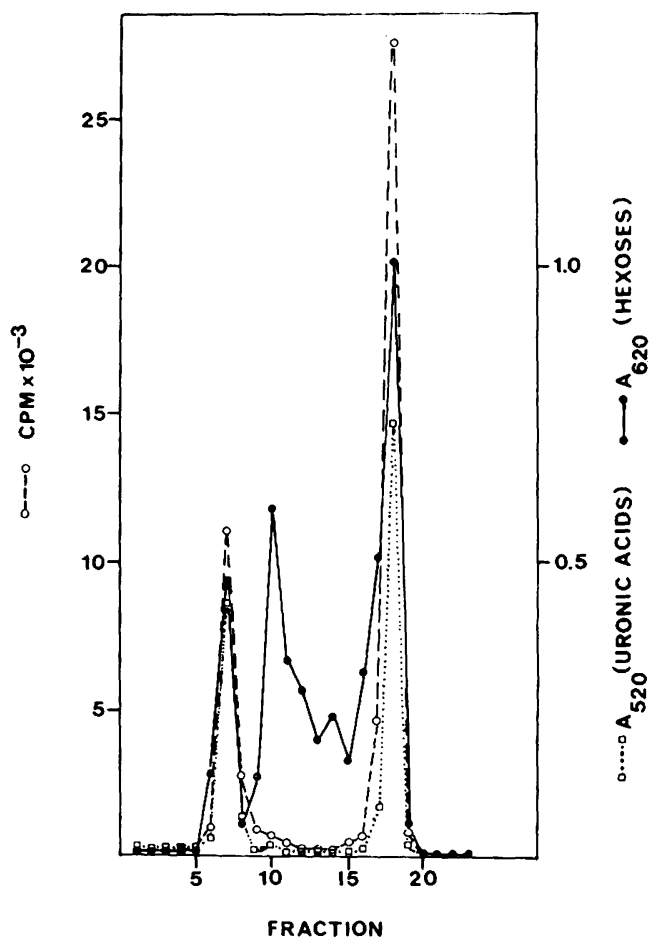

Fig. 2. Separation of glycopeptides derived from pronase digestion of ${ }^{35} \mathrm{SO}_{4}{ }^{2-}$-labelled Halobacterial cell wall glycoprotein by chromatography on Bio Gel P 10.

Column: $1.6 \times 80 \mathrm{~cm}$; buffer pyridine/acetate, $0.1 \mathrm{M}$. pH 5.5. $\bigcirc-. . .-\mathrm{O}$ radioactivity, method).

weight saccharide containing amino sugars will be referred to in the following as HMW-saccharide.

An additional radioactive peak is observed eluting in a volume corresponding to a low molecular weight. This fraction again contains neutral sugars and uronic acids, but no amino sugars are found. Eluting between the two radioactive peaks are glycopeptides which contain solely neutral sugars. The compositions of the three fractions are given in Table 1.

\section{The $H M W$-saccharide has a repeating unit structure}

The ${ }^{35} \mathrm{SO}_{4}{ }^{2-}$-labelled $\mathrm{HMW}$-saccharide fraction on high voltage thin layer electrophoresis shows one single spot (c. f. Fig. $8 \mathrm{Aa}$ ). On the other hand, on SDS gel electrophoresis (Laemmli, 1970) or polyacrylamide gel electrophoresis in the presence of urea (Maxam and Gilbert, 1977) this fraction exhibits a striking regular pattern of about 15 bands (Fig. $3 \mathrm{~A}$ and Fig. $4 \mathrm{~A}$ ). This pattern, which is similar to that of oligonucleotides of different chain lengths on polyacrylamide gel electrophoresis, led us to the idea that the different bands represent different 
Table 1. Composition of three different fractions obtained after pronase digestion of ${ }^{35} \mathrm{SO}_{4}{ }^{2-}$-labelled cell wall glycoprotein and chromatography on Bio Gel P 10

\begin{tabular}{llllllll}
\hline & Glc & Gal & GlcUA & GalUA & GlcN & GalN & SO $_{4}{ }^{2-}$ \\
\hline $\begin{array}{l}\text { HMW-saccharides } \\
\quad \text { (void volume) }\end{array}$ & - & 1 & - & 2 & 1 & 1 & 2 \\
$\begin{array}{c}\text { Neutral-saccharides } \\
\quad \text { (middle molecular }\end{array}$ & 1 & 1 & - & - & - & - & - \\
$\quad$ weight range) \\
$\begin{array}{l}\text { Low molecular weight } \\
\text { saccharides }\end{array}$
\end{tabular}

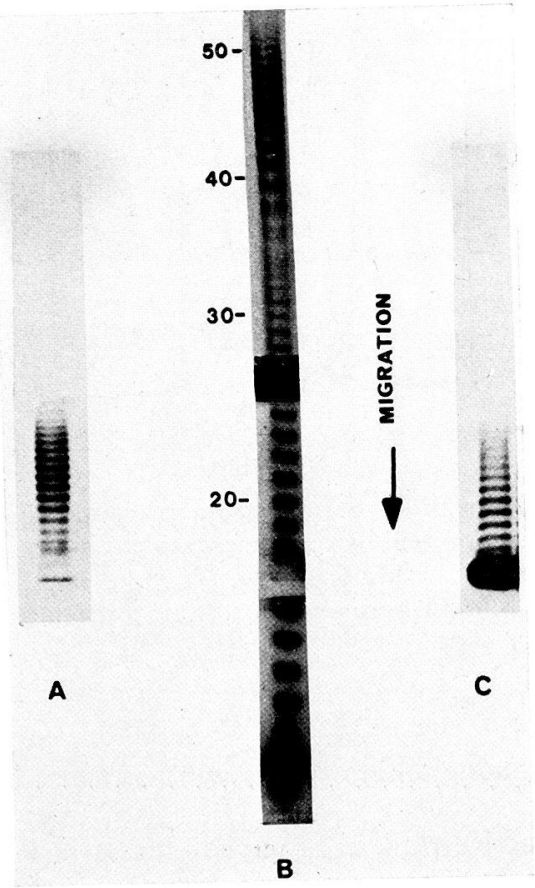

Fig. 3. Analyses of ${ }^{35} \mathrm{SO}_{4}{ }^{2-}$-labelled HMW-saccharides by electrophoresis on a SDS gel (A) and on a urea containing polyacrylamide gel according to Maxam and Gilbert (1977). For estimation of the ${ }^{35} \mathrm{SO}_{4}{ }^{2-} \mathrm{HMW}$-saccharides' average chain length a sample was mixed with ${ }^{32} \mathrm{PO}_{4}{ }^{3-}$-labelled oligoribonucleotides of different chain lengths before electrophoresis. The numbers indicate ribonucleotide units (B). SDS-gel electrophoresis of a total cell lysate after in vivo labelling of Halobacteria with ${ }^{35} \mathrm{SO}_{4}{ }^{2-}$ in the presence of ethidium bromide (C). The radioactive material is visualized by fluorography.

chain lenghts of a repeating sulphated saccharide unit. In order to examine this suggestion, three homogeneous glycopeptides far distant from each other were isolated from a gel to compare their fragmentation patterns. This was performed by mild acid hydrolysis of the isolated homogeneous HMW-saccharides and subsequent high voltage thin layer electrophoresis (c. f. Fig. 4): the total mixture of 
A

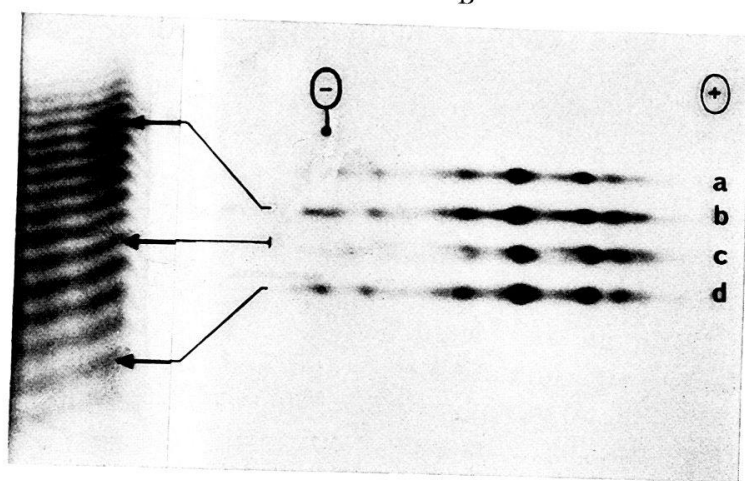
Fig. 4 . Comparison of the fragmentation patterns of isolated homogeneous HMW-sac-
charides.

Radioactive material was eluted from a Maxam and Gilbert gel as marked with arrows (A). The isolated $\mathrm{HMW}$-saccharides were incubated with $0.25 \mathrm{~N} \mathrm{HCl}$ for $15 \mathrm{~min}$ at $100{ }^{\circ} \mathrm{C}$ and subsequently subjected to high voltage thin layer electrophoresis at $\mathrm{pH} 2.0$. Fluorogram after the run (B): total ${ }^{35} \mathrm{SO}_{4}{ }^{2-} \mathrm{HMW}$-saccharides mixture (a), isolated HMW-saccharides (b to d).

HMW-saccharides as well as the isolated homogeneous samples all gave rise to an identical fragmentation pattern. This is taken as strong evidence for a repetitive sequence structure of the HMW-saccharides. Further proof for a repeating unit structure comes from separation and analysis of fragmentation products after

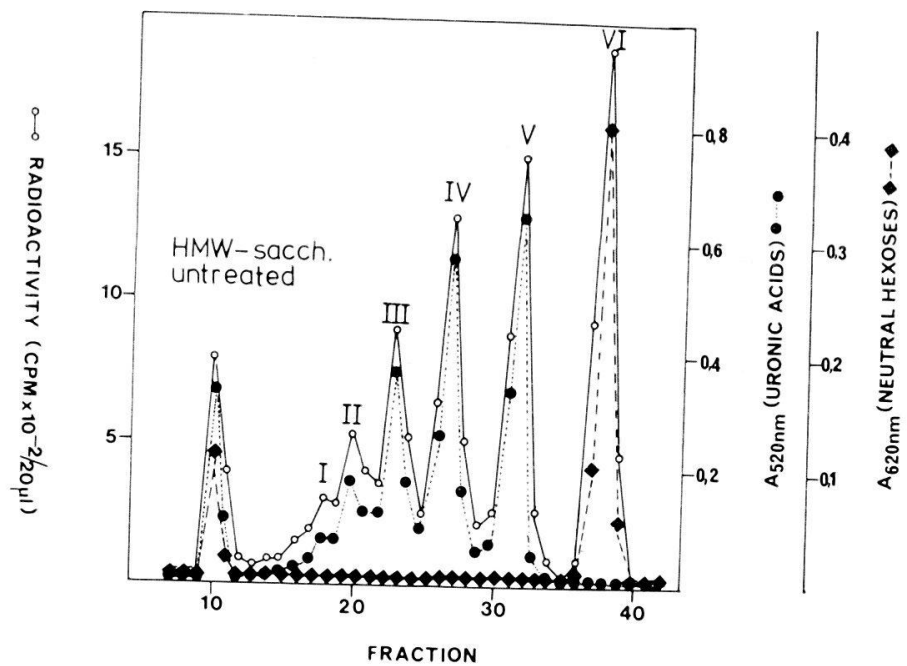

Fig. 5. Separation of HMW-saccharide fragments according to their molecular weights. Conditions as under Fig. 2. To a sample after mild acid hydrolysis (c.f. legend to Fig. untreated ${ }^{35} \mathrm{SO}_{4}{ }^{2-} \mathrm{HMW}$-saccharide was added as an internal molecular weight standard. The mixture was chromatographed on Bio Gel P 10 . $\bigcirc-\ldots$ radioactivity, uronic acids, neutral hexoses. 
graded acid hydrolysis of the HMW-saccharide fraction: a ${ }^{35} \mathrm{SO}_{4}{ }^{2-}$-labelled sample was incubated for $15 \mathrm{~min}$ at $100^{\circ} \mathrm{C}$ with $0.25 \mathrm{~N} \mathrm{HCl}$ and subsequently chromatographed on a Bio Gel P 10 column (untreated ${ }^{35} \mathrm{SO}_{4}{ }^{2-} \mathrm{HMW}$-saccharides were included as an internal molecular weight standard): as can be seen in Fig. 5 mild acid hydrolysis gives rise to six new peaks of different molecular weights. Peaks I to $\mathrm{V}$ all contain sulphate, galacturonic acid and amino sugars at a constant ratio, whereas peak VI contains only galactose and free sulphate. Thus the repetitive saccharide unit possibly consists of the amino sugars, galacturonic acids and sulphate residues with a peripherally bound galactose residue. Work is in progress to elucidate this structure in more detail.

By comparative polyacrylamide gel electrophoresis the average chain length of the HMW-saccharides (band of highest ${ }^{35} \mathrm{SO}_{4}{ }^{2-}$ intensity) is found to be equivalent to 45 ribonucleotide units (Fig. $3 \mathrm{~B}$ ).

\section{The HMW-saccharide has a form-keeping function}

Halobacteria grown in the presence of the antibiotic bacitracin are no longer rod-shaped but quantitatively convert to regular spheres (Mescher and Strominger, 1975). In addition these authors reported that the overall carbohydrate content was somewhat diminished under the influence of bacitracin. We found that in the presence of bacitracin a sulphated cell wall glycoprotein is synthesized, which differs from the normal sulphated cell wall glycoprotein by a small increase in mobility on SDS-gels, indicating a lower molecular weight. To investigate the structural difference between the normal ${ }^{35} \mathrm{SO}_{4}{ }^{2-}$-labelled cell wall glycoprotein and the ${ }^{35} \mathrm{SO}_{4}{ }^{2-}$-glycoprotein from Halobacteria labelled in the presence of bacitracin, pronase glycopeptides were analyzed by high pressure liquid chromatography on an anion exchange column: Fig. $6 \mathrm{~A}$ represents the radioactivity profile of ${ }^{35} \mathrm{SO}_{4}{ }^{2-}$-glycopeptides from normal cell wall glycoprotein. Peaks a to i do not contain amino sugars and therefore represent the sulphated low molecular weight saccharides, whereas peak $\mathrm{z}$ is caused by the HMW-saccharides fraction. Separation of the glycopeptides derived from glycoprotein of cells labelled in the presence of bacitracin is shown in Fig. 6B: the sample completely lacks the HMW-saccharide peak. From the absence of the HMW-saccharide in spherical cells we conclude that it is the function of this glycoconjugate to maintain the rod form to Halobacteria.

\section{The HMW-saccharide is synthesized via a lipid linked precusor}

The inhibition by bacitracin of the incorporation of the repeating unit saccharide into the glycoprotein as well as the suggestion that lipid intermediates are involved in the biosynthesis of the amino sugar containing saccharide (Mescher and Strominger, 1978) lead us to search for a lipid linked precursor of this saccharide. For this purpose Halobacteria were labelled in vivo with ${ }^{35} \mathrm{SO}_{4}{ }^{2-}$ in the presence of ethidium bromide in a concentration inhibitory for protein synthesis. Analysis of total cell lysates by SDS-gel electrophoresis yielded a striking regular pattern of bands similar to that obtained by exhaustive digestion of the isolated ${ }^{35} \mathrm{SO}_{4}{ }^{2-}$ labelled cell wall glycoprotein (Fig. $3 \mathrm{C}$ ).

Attempts to isolate this material revealed that the radioactivity copurified with the membrane. After sucrose density gradient centrifugation the material in question was quantitatively recovered in the membrane fraction (Fig. $7 \mathrm{~A}$ ) indicating 

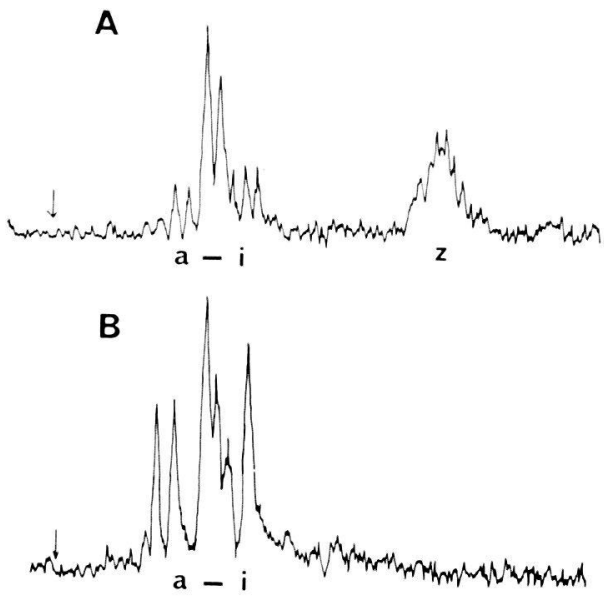

Fig. 6. Spherical Halobacteria lack the HMW-saccharide fraction. Radioactivity profile of ${ }^{35} \mathrm{SO}_{4}{ }^{2-}$-labelled glycopeptides from cell wall glycoprotein after separation by high pressure liquid chromatography from rod shaped cells (a) and from spherical cells grown in the
presence of bacitracin (B).

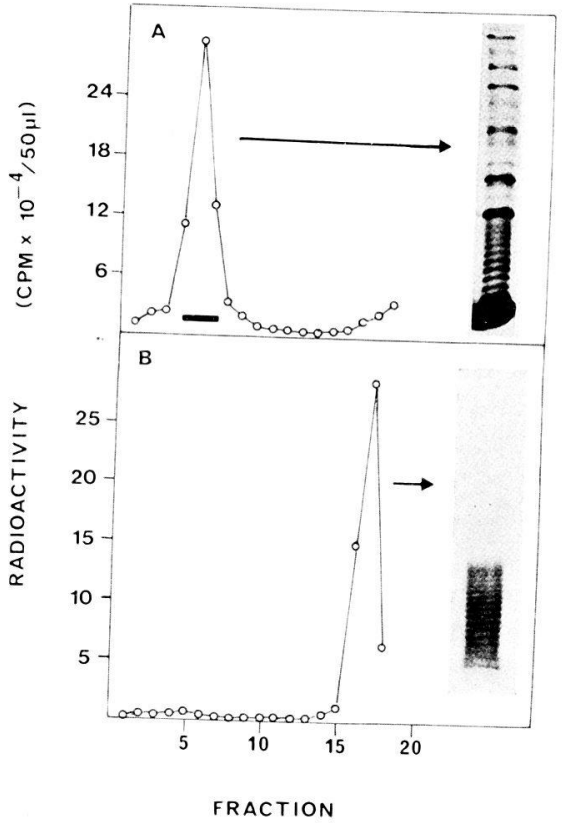

Fig. 7. Sucrose density gradient centrifugation of a crude membrane fraction of Halobacteria after in vivo labelling with ${ }^{35} \mathrm{SO}_{4}{ }^{2-}$ (A) and of a mixture of an unlabelled crude membrane fraction with an isolated ${ }^{35} \mathrm{SO}_{4}{ }^{2-} \mathrm{HMW}$-saccharide fraction as a control (B). The inserts show SDS-gels of the corresponding peak fractions. 
its lipid character. The material is best solubilized with aqueous propanol after delipidation of the purified membranes with chloroform/methanol. It is completely insoluble in aqueous buffers. To investigate the suggested structural relationship of the lipid-bound radioactive material and the ${ }^{35} \mathrm{SO}_{4}{ }^{2-}-\mathrm{HMW}$-saccharides, the pattern obtained after subjection to different fragmentation methods were compared. Fig. 8 shows a high voltage thin layer electrophoresis after mild acid hydrolysis of the membrane - bound radioactive material (c) and of the HMW-saccharides (d). As mentioned above untreated HMW-saccharides migrate as an single spot (a), whereas untreated lipid linked material due to its lipid character does not migrate at all in the aquous buffer system used (b). Identity of the saccharide part of both materials is confirmed by the pattern obtained after hydrazinolysis and nitrous acid deamination (Fig. $8 \mathrm{~B}$, a to $\mathrm{c}$ ).

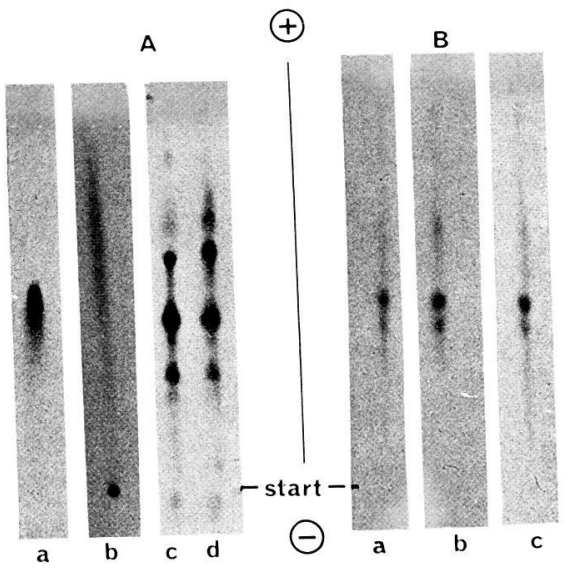

Fig. 8. The ${ }^{35} \mathrm{SO}_{4}{ }^{2-}$ lipid-linked material is a precursor of the $\mathrm{HMW}$-saccharide.

Isolated ${ }^{35} \mathrm{SO}_{4}{ }^{2-}$ lipid-linked material and ${ }^{35} \mathrm{SO}_{4}{ }^{2-} \mathrm{HMW}$-saccharides after fragmention by different methods were subjected to high voltage thin layer electrophoresis. A: ${ }^{35} \mathrm{SO}_{4}{ }^{2-}$ labelled $\mathrm{HMW}$-saccharides (a) and ${ }^{35} \mathrm{SO}_{4}{ }^{2-}$-labelled lipid-linked material (b), untreated. The samples after mild acid hydrolysis (c) and (d), respectively. B : after hydrazinolysis and nitrous acid deamination. HMW-saccharides (a), mixture of HMW-saccharides and lipidlinked material (b), lipid linked material (c).

\section{Discussion}

Fingerprint analyses according to Cleveland et al. (1977) demonstrated identity of the cell wall glycoproteins from Halobacterium halobium and Halobacterium salinarium. In addition, ${ }^{35} \mathrm{SO}_{4}{ }^{2-}$ is incorporated into the Halobacterium salinarium glycoprotein as well, and the fragmentation patterns of both radioactive glycoproteins were identical (Dompert and Sumper, unpublished). Therefore our data may be compared with the results on the Halobacterium salinarium cell wall glycoprotein.

In agreement with Mescher and Strominger (1976) we found a neutral disaccharide linked to the cell wall glycoprotein which contained glc and gal. We were not able, however, to find trisaccharides containing glc, gal and an uronic acid. On the other hand there was a glycopeptide fraction containing glc, glcUA and 
sulphate. Investigations concerning the nature of the linkage and the structure of these saccharides are in progress.

The amino sugar containing HMW-saccharide in our hands showed quite a different composition than that described for the amino sugar containing Halobacterium salinarium saccharide: we found neither man nor glc but galacturonic acid and sulphate which occur in appreciable amounts in the HMW-saccharides (c. f. Table 1). From the identity of the fragmentation patterns of HMW-saccharides of different molecular weights (c. f. Fig. 4) and from the composition of isolated fragments of different molecular weights (c.f. Fig. 5) we conclude that the so called "heterosaccharide" is a glycoconjugate which is made up by a sulphated repeating unit: a structure which is similar to that of the animal glycosaminoglycans. The about 15 bands found on SDS gels of a ${ }^{35} \mathrm{SO}_{4}{ }^{2-}$-labelled HMWsaccharide fraction thus represent about 15 different chain lengths of the repeating unit saccharide, the distance between two adjacent bands being due to one repeating unit.

From the finding that Halobacterial spheres grown in the presence of bacitracin completely lack the HMW-saccharide fraction we conclude that this glycosaminoglycan-like structure is involved in maintaining the rod form of normal Halobacteria.

The biosynthesis of this sulphated repeating unit saccharide seems to be different from that known for the glycosaminoglycans (for a review see Rodén, 1980): the saccharide chain is not elongated step by step on a protein bound linker region, but the HMW-saccharides are synthesized on and transferred from a lipid carrier. Remarkably, the sulphate residues are detectable already in the lipid linked precursor at all degrees of polymerization. This again seems to be unusual, as in the synthesis of heparin, for example, sulphate residues are introduced into the molecule only after glycosidic polymerisation is finished (Lindhal and Höok, 1978).

The lipid linked to the HMW-saccharide precursor according to Mescher et al. (1976) and Mescher and Strominger (1978) most probably is a polyprenyl of 11 to 12 isoprene units. Solubility of the precursor in a solvent more hydrophilic (propanol/water, 1/1) than the solvents normally used for polyprenyl-sugar compounds $\left(\mathrm{CHCl}_{3} / \mathrm{CH}_{3} \mathrm{OH} / \mathrm{H}_{2} \mathrm{O}=10 / 10 / 3\right.$, for example $)$ is easily explained by the presence of negative charges introduced by the sulphate residues.

We could not yet ultimately decide whether the observed heterogeneity in chain length of the HMW-saccharide is caused artificially by the very low sulphate concentration necessary in ${ }^{35} \mathrm{SO}_{4}{ }^{2-}$-labelling experiments or whether it simply reflects a relatively broad chain length specificity of the corresponding transferase. The latter is probably true, as the pattern on SDS gels of the HMW-saccharides is not influenced by varying the sulphate concentration in ${ }^{35} \mathrm{SO}_{4}{ }^{2-}$-labelling experiments between about $7 \cdot 10^{-6} \mathrm{M}$ to $5 \cdot 10^{-3} \mathrm{M}$.

Acknowledgment. This work was supported by the Deutsche Forschungsgemeinschaft (SFB 43, Regensburg).

\section{References}

Cleveland, D.W., Fischer, S.G., Kirschner, M.W., Laemmli, K.K.: Peptide Mapping by Limited Proteolysis in Sodium Dodecyl Sulfate and Analysis by Gel Electrophoresis. J. Biol. Chem. 252, 1102-1106 (1977) 
Laemmli, U.K.: Cleavage of structural proteins during the assembly of the head of bacteriophage $T_{.}$. Nature (Lond.) 227, 680-685 (1970)

Lindahl, U., Höok, M.: Glycosaminoglycans and their Binding to Biological Macromolecules. Ann. Rev. Biochem. 47, 385-417 (1978)

Maxam, A.M., Gilbert, W.: A new method for sequencing DNA. Proc. nat. Acad. Sci. (Wash.) 74, 560-564 (1977)

Mescher, M.F., Strominger, J.L., Watson, S. W.: Protein and Carbohydrate Composition of the Cell Envelope of Halobacterium salinarium. J. Bact. 120, 945-954 (1974)

Mescher, M.F., Strominger, J.L.: Bacitracin Induces Sphere Formation in Halobacterium Species which Lack a Wall Peptidoglycan. J. gen. Microbiol. 89, 375-378 (1975)

Mescher, M.F., Strominger, J.L.: Purification and Characterization of a Procariotic Glycoprotein from the Cell Envelope of Halobacterium salinarium. J. Biol. Chem. 251, 20052014 (1976)

Mescher, M.F., Hansen, U., Strominger, J.L.: Formation of Lipid-linked Sugar Compounds in Halobacterium salinarium. J. Biol. Chem. 251, 7289-7294 (1976)

Mescher, M.F., Strominger, J.L.: Glycosilation of the Surface Glycoprotein of Halobacterium salinarium via a Cyclic Pathway of Lipid-linked Intermediates. FEBS Lett. 89, 37-41 (1978)

Rodén, L.: Structure and Metabolism of Connective Tissue Proteoglycans. In: The Biochemistry of Glycoproteins and Proteoglycans (W.L.Lennarz, ed.). New York, Plenum Press, 1980

Sumper, M., Herrmann, G.: Studies on the Biosynthesis of Bacterioopsin. Europ. J. Biochem. 89, 229-235 (1978)

Wieland, F., Dompert, W., Bernhardt, G., Sumper, M.: Halobacterial Glycoprotein Saccharides Contain Covalently Linked Sulphate. FEBS Lett. 120, 110-114 (1980)

Wieland, F., Lechner, J., Bernhardt, G., Sumper, M.: Sulphation of a Repetitive Saccharide in Halobacterial Cell Wall Glycoprotein: Occurrence of a Sulphated Lipid-linked Precursor. FEBS Lett., in the press (1981)

Dr. Felix Wieland, Lehrstuhl für Biochemie I der Universität, Universitätsstr., D-8400 Regensburg, FRG 